\title{
GENERALIZED COMMUTATORS AND THE MOORE-PENROSE INVERSE*
}

\author{
IRWIN S. PRESSMAN ${ }^{\dagger}$
}

\begin{abstract}
This work studies the kernel of a linear operator associated with the generalized k-fold commutator. Given a set $\mathfrak{A}=\left\{A_{1}, \ldots, A_{k}\right\}$ of real $n \times n$ matrices, the commutator is denoted by $\left[A_{1}|\ldots| A_{k}\right]$. For a fixed set of matrices $\mathfrak{A}$ we introduce a multilinear skew-symmetric linear operator $T_{\mathfrak{A}}(X)=T\left(A_{1}, \ldots, A_{k}\right)[X]=\left[A_{1}|\ldots| A_{k} \mid X\right]$. For fixed $n$ and $k \geq 2 n-1, T_{\mathfrak{A}} \equiv 0$ by the Amitsur-Levitski Theorem [2], which motivated this work. The matrix representation $M$ of the linear transformation $T$ is called the k-commutator matrix. $M$ has interesting properties, e.g., it is a commutator; for $k$ odd, there is a permutation of the rows of $M$ that makes it skew-symmetric. For both $k$ and $n$ odd, a provocative matrix $\mathcal{S}$ appears in the kernel of $T$. By using the Moore-Penrose inverse and introducing a conjecture about the rank of $M$, the entries of $\mathcal{S}$ are shown to be quotients of polynomials in the entries of the matrices in $\mathfrak{A}$. One case of the conjecture has been recently proven by Brassil. The Moore-Penrose inverse provides a full rank decomposition of $M$.
\end{abstract}

Key words. Generalized commutator, Amitsur-Levitski Theorem, Moore-Penrose inverse.

AMS subject classifications. 15A04, 15A09, 47B47.

1. The generalized commutator $\left[A_{1}\left|A_{2}\right| \ldots \mid A_{k}\right]$. We denote the space of $n \times n$ matrices over the real numbers by $\mathcal{M}_{n}(\mathbb{R})$ or $\mathcal{M}_{n}$. Define the generalized commutator [10] ${ }^{1}$ inductively as follows. Choose $A_{i} \in \mathcal{M}_{n}, i=1,2, \ldots, k$, and let $\mathfrak{A}$ denote the set $\left\{A_{1}, A_{2}, \ldots, A_{k}\right\}$. For $k=0,[]:=I$, for $k=1\left[A_{1}\right]:=A_{1}$. In general for $k \geq 1$ we have

$$
\left[A_{1}|\ldots| A_{k}\right]=\sum_{i=1}^{k}(-1)^{i+1} A_{i} C_{i}
$$

where $C_{i}:=\left[A_{1}|\ldots| \widehat{A_{i}}|\ldots| A_{k}\right]$ is the generalized commutator of $k-1$ matrices (where the "hat" symbol indicates omitting $A_{i}$ from the list of inputs). Induction shows that

$$
\left[A_{1}|\ldots| A_{k}\right]=\sum_{\pi} \operatorname{sgn}(\pi) A_{\pi(1)} \ldots A_{\pi(k)}
$$

where the sum is over all permutations $\pi$ of $[1,2, \ldots, k]$, and (1.2) is called the standard polynomial [17, 2]. Studies of identities relating to this commutator have been made in Algebra [2, 3, 8] , Lie Algebras [12], and Physics [9].

*Received by the editors on March 28, 2019. Accepted for publication on September 1, 2021. Handling Editor: Bryan Shader.

${ }^{\dagger}$ School of Mathematics and Statistics, Carleton University, 1125 Colonel By Drive, Ottawa Ontario, CANADA K1S 5B6 ORCID 0000-0003-3162-7212.

¥I gratefully acknowledge that this research was supported by the Fields Institute for Research in Mathematical Sciences. Its contents are solely the responsibility of the author and do not necessarily represent the official views of the Institute.

${ }^{1}$ These commutators were called $N$-commutators by Dzhumadil'daev [12] in studies of Lie Algebras. They were called higher order brackets or multibrackets and used to study Generalized Lie algebras and n-ary Algebras in [4]. In the case $n=3$ they are called ternary commutators [8], or ternutators [9]. 
REMARK 1.1.

(i) The generalized commutator is linear in each of its arguments and is an alternating function (Amitsur-Levitski, [2]).

(ii) $\left[A_{1}|\ldots| A_{k}\right]$ is the zero matrix if any two arguments are equal.

Proposition 1.2. Let $r$ and $n$ be positive integers with $1 \leq r \leq n$, and let $A_{1}, \ldots, A_{n}$ be $n \times n$ matrices with $A_{r}=I$. Then

$$
\left[A_{1}\left|A_{2}\right| \cdots \mid A_{n}\right]= \begin{cases}0 & \text { if } n \text { is even } \\ (-1)^{r-1}\left[A_{1}\left|A_{2}\right| \cdots\left|\widehat{A_{r}}\right| \cdots \mid A_{n}\right] & \text { if } n \text { is odd. }\end{cases}
$$

Proof. Consider the case where $n$ is even. Pair the entries of each term of the commutator. Take $\tilde{A}$ a particular matrix other than $I$ in the set $\mathfrak{A}$. Every occurrence in the expansion (1.1) of the commutator where the "twosome" $\bullet I_{n} \tilde{A} \bullet$ are together, can be matched with a unique term $\bullet \tilde{A} I_{n} \bullet$, where all other entries are identical. These occur with opposite signature, so all product terms cancel in pairs. $\left[\bullet \bullet \vdots \bullet \vdots \ldots \vdots I_{n} \tilde{A}: \bullet\right.$ $\bullet \vdots . .$.$] cancels \left[\bullet \bullet \bullet \bullet \vdots \ldots \vdots \tilde{A} I_{n} \vdots \bullet \bullet \ldots . \ldots\right]$.

Consider the case where $n$ is odd, and let $A_{r}=I$. By the above result, each term $C_{i}=0$ in equation (1.1) unless $i=r$, since $C_{i}$ would otherwise have an identity matrix entry. The result follows at once.

We next observe that a similar set of input matrices produces a similar commutator.

Proposition 1.3. For $Q$ nonsingular and matrices $\left\{A_{1}, A_{2}, \ldots, A_{k}\right\}$ all in $\mathcal{M}_{n}$ and $C=\left[A_{1}|\ldots| A_{k}\right]$, then $\left[Q^{-1} A_{1} Q\left|Q^{-1} A_{2} Q\right| \ldots \mid Q^{-1} A_{k} Q\right]=Q^{-1} C Q$.

Proof. $\left[Q^{-1} A_{1} Q|\ldots| Q^{-1} A_{k} Q\right]=\sum_{\pi} \operatorname{sgn}(\pi) Q^{-1} A_{\pi(1)} Q Q^{-1} A_{\pi(2)} Q Q^{-1} A_{\pi(3)} Q \ldots Q^{-1} A_{\pi(k)} Q$ $=\sum_{\pi} \operatorname{sgn}(\pi) Q^{-1} A_{\pi(1)} A_{\pi(2)} A_{\pi(3)} \ldots A_{\pi(k)} Q=Q^{-1} C Q$.

A complete directed graph $\Delta_{n}$ has vertices $\{1,2, \ldots, n\}$ and to every ordered pair of vertices $i, j$ there is a unique directed $\operatorname{arc} i \rightarrow j$. There are loops $i \rightarrow i$.

Definition 1.4. A trail is a walk in $\Delta_{n}$ without repeated edges. We omit the $n$ subscript on $\Delta$ when the size is clear.

Trails are instrumental in Swan's proof of the Amitsur-Levitski Theorem [18]. A non-zero element in the $(i, j)$ position of the commutator $C=\left[A_{1}|\ldots| A_{k}\right]$ consists of the sum of all expressions of the form

$$
\operatorname{sgn}(\pi) a_{i, i_{2}}^{\pi_{1}} a_{i_{2}, i_{3}}^{\pi_{2}} \ldots a_{i_{k-1}, j}^{\pi_{k}},
$$

where $a_{i_{t}, i_{t+1}}^{\pi_{t}}$ is in the $\pi_{t}^{t h}$ matrix, for each permutation $\pi$ of $1, \ldots, k$ and corresponding to a trail in $\Delta$ of length $k$ from $i$ to $j$. For example, given four $3 \times 3$ matrices $\mathcal{F}_{4}=\{A, B, C, D\}$ the trail $2 \rightarrow 3 \rightarrow 2 \rightarrow 1 \rightarrow 1$ produces 24 elements in the $(2,1)$ position of the commutator $[A|B| C \mid D]$.

$$
\begin{aligned}
& +a_{23} b_{32} c_{21} d_{11}-a_{23} b_{32} d_{21} c_{11}+a_{23} c_{32} d_{21} b_{11}-a_{23} d_{32} c_{21} b_{11} \\
& +a_{23} d_{32} b_{21} c_{11}-a_{23} d_{32} c_{21} b_{11}+b_{23} a_{32} d_{21} c_{11}-b_{23} a_{32} c_{21} d_{11} \\
& +b_{23} c_{32} a_{21} d_{11}-b_{23} c_{32} d_{21} a_{11}+b_{23} d_{32} c_{21} a_{11}-b_{23} d_{32} a_{21} c_{11} \\
& +c_{23} a_{32} b_{21} d_{11}-c_{23} a_{32} d_{21} b_{11}+c_{23} b_{32} d_{21} a_{11}-c_{23} d_{32} b_{21} a_{11} \\
& +c_{23} d_{32} a_{21} b_{11}-c_{23} d_{32} b_{21} a_{11}+d_{23} b_{32} a_{21} c_{11}-d_{23} b_{32} c_{21} a_{11} \\
& +d_{23} a_{32} c_{21} b_{11}-d_{23} c_{32} a_{21} b_{11}+d_{23} c_{32} b_{21} a_{11}-d_{23} c_{32} a_{21} b_{11}
\end{aligned}
$$


By exhaustive search, we find 40 trails from 2 to 1 of length 4 that contribute to the $(2,1)$ position of the commutator $[A|B| C \mid D]$. We consistently denote the transpose of a matrix $B \in \mathcal{M}_{n}$ by $B^{\prime}$.

Definition 1.5. For an $n \times n$ matrix $H$ define vec $(H)$ to be the $n^{2}$-column vector obtained by stacking successive columns $H_{1}, \ldots, H_{n}$ of $H$. For a $3 \times 3$ matrix $H=\left\{h_{i, j}\right\}$, the transpose vec $(H)^{\prime}=\left[h_{11}, h_{21}, h_{31}\right.$, $\left.h_{12}, h_{22}, h_{32}, h_{13}, h_{23}, h_{33}\right]$, is called a canonical row. Following MATLAB(C) notation, the operation inverse to the vec operation is reshape.

$$
\text { Use the set } \mathcal{F}_{4} \text { to construct the } 4 \times n^{2} \text { matrix } V_{4}=\left[\begin{array}{c}
\operatorname{vec}(A)^{\prime} \\
\operatorname{vec}(B)^{\prime} \\
\operatorname{vec}(C)^{\prime} \\
\operatorname{vec}(D)^{\prime}
\end{array}\right] \text {. }
$$

The above term (1.4) is the determinant of the submatrix of $V_{4}$ using the $8^{\text {th }}, 6^{\text {th }}, 2^{\text {nd }}, 1^{\text {st }}$ columns of the $4 \times 9 V_{4}$ matrix, in the given order, corresponding to the trail $2 \rightarrow 3 \rightarrow 2 \rightarrow 1 \rightarrow 1$. Each element of the commutator is given by sums of determinants corresponding to trails!

LEMMA 1.6. Each of the following is a sufficient condition for $\left[A_{1}|\ldots| A_{k}\right]$ to be 0

(i) Two of the matrices $A_{1}, \ldots, A_{k}$ are equal.

(ii) The matrices $A_{1}, \ldots, A_{k}$ are linearly dependent over $\mathbb{R}$.

(iii) $k$ is even and the matrices $A_{1}, \ldots, A_{k}, I_{n}$ are linearly dependent over $\mathbb{R}$.

(iv) $\mathfrak{A}=\left\{A_{1}, \ldots, A_{k}\right\}$ is a commuting set of matrices.

Proof.

(i) Since each of the matrices in question has a pair of equal rows, the determinants are 0 for each trail. See also Remark 1.1 and [2].

(ii) If $A_{i}$ is a linear combination of $A_{1}, \ldots, A_{i-1}$ then use multilinearity to expand $\left[A_{1}|\ldots| A_{k}\right]$ and obtain a linear combination of generalized commutators in which the $i^{\text {th }}$ entry is replaced by a linear combination of the previous matrices. The result follows by (i). In particular, $\left[A_{1}|\ldots| A_{k} \mid A_{j}\right]=0$ for every $j, 1 \leq j \leq k$.

(iii) This follows from the linearity of the generalized commutator, (ii) and Proposition 1.2.

(iv) Every term in the expansion (1.2) of the commutator is equal except for the sign of the permutation. These cancel in pairs.

2. The operator $T: X \longmapsto\left[A_{1}|\ldots| A_{k} \mid X\right]$ and the k-commutator matrix $M$ for $T$. Choose a set $\mathfrak{A}=\left\{A_{1}, A_{2}, \ldots, A_{k}\right\}$. The set of all elementary matrices $E_{i, j}$ with a 1 in the $(i, j)$ position and 0 elsewhere give the standard basis of the vector space $\mathcal{M}_{n}(\mathbb{R})$. A canonical ordering for the standard basis is given by

$$
E_{1,1}, E_{2,1}, E_{3,1}, \ldots, E_{n, 1}, E_{1,2}, E_{2,2}, E_{3,2} \ldots, E_{n, 2}, E_{1,3}, \ldots E_{1, n}, \ldots, E_{n, n} .
$$

Given $A_{1}, \ldots, A_{k}, X \in \mathcal{M}_{n}, X=\sum_{i=1}^{n} \sum_{j=1}^{n} x_{i, j} E_{i, j}$ define the linear operator

$$
T: X \longmapsto\left[A_{1}|\ldots| A_{k} \mid X\right] \text {. }
$$

The generalized commutator is linear in $x_{i, j}$ so

$$
\begin{aligned}
\operatorname{vec}\left(\left[A_{1}|\ldots| A_{k} \mid X\right]\right) & =\operatorname{vec}\left(\left[A_{1}|\ldots| A_{k} \mid \sum_{i=1}^{n} \sum_{j=1}^{n} x_{i, j} E_{i, j}\right]\right) \\
& =\sum_{i=1}^{n} \sum_{j=1}^{n} x_{i, j} \operatorname{vec}\left(\left[A_{1}|\ldots| A_{k} \mid E_{i, j}\right]\right)=\operatorname{Mvec}(X)
\end{aligned}
$$


Electronic Journal of Linear Algebra, ISSN 1081-3810

A publication of the International Linear Algebra Society

Volume 37, pp. 598-612, September 2021.

where $M=M_{\mathfrak{A}}$ is the matrix generated by $\mathfrak{A}$ with columns $\operatorname{vec}\left(\left[A_{1}|\ldots| A_{k} \mid E_{i, j}\right]\right)$ in the canonical order (2.5), and is called the $k$-commutator matrix for $T$ with respect to the standard basis. ${ }^{2}$

Remark 2.1. By Remark 1.1, for $1 \leq j \leq k, T\left(A_{j}\right)=O_{n}$, the $n \times n$ zero matrix, and $M v e c\left(A_{j}\right)=$ $0_{n^{2}}$, the $n^{2} \times 1$ zero vector. By Proposition 1.2, for $k$ odd, $T\left(I_{n}\right)=O_{n}$ and $M v e c\left(I_{n}\right)=0_{n^{2}}$. Let $\mathcal{U}=$ $\left\{A_{1}, \ldots, A_{2 m+1}\right\}$ and $\mathcal{U}^{*}=\left\{A_{1}, \ldots, A_{2 m+1}, I\right\}$. Proposition 1.2 indicates that $M_{\mathcal{U}}=M_{\mathcal{U}^{*}}$. By the AmitsurLevitski Theorem $[2,13], T \equiv O$ if $k \geq 2 n-1$ and $k=2 n$ is the degree of the minimal (non-commutative) polynomial (1.2).

The $(i+(n-1) j)^{t h}$ column of $M$ is $\operatorname{vec}\left(\left[A_{1}|\ldots| A_{k} \mid E_{i, j}\right]\right)$.

2.1. The Null Space of $M$. The properties of the matrix $M$ of the linear transformation $T$ are explained, for $n$ odd, by permutations $P^{\diamond}$ introduced here. An example is given here.

EXAMPLE 2.2.

$$
P_{4}^{\diamond}=\left[\begin{array}{llllllllllllllll}
1 & 0 & 0 & 0 & 0 & 0 & 0 & 0 & 0 & 0 & 0 & 0 & 0 & 0 & 0 & 0 \\
0 & 0 & 0 & 0 & 1 & 0 & 0 & 0 & 0 & 0 & 0 & 0 & 0 & 0 & 0 & 0 \\
0 & 0 & 0 & 0 & 0 & 0 & 0 & 0 & 1 & 0 & 0 & 0 & 0 & 0 & 0 & 0 \\
0 & 0 & 0 & 0 & 0 & 0 & 0 & 0 & 0 & 0 & 0 & 0 & 1 & 0 & 0 & 0 \\
0 & 1 & 0 & 0 & 0 & 0 & 0 & 0 & 0 & 0 & 0 & 0 & 0 & 0 & 0 & 0 \\
0 & 0 & 0 & 0 & 0 & 1 & 0 & 0 & 0 & 0 & 0 & 0 & 0 & 0 & 0 & 0 \\
0 & 0 & 0 & 0 & 0 & 0 & 0 & 0 & 0 & 1 & 0 & 0 & 0 & 0 & 0 & 0 \\
0 & 0 & 0 & 0 & 0 & 0 & 0 & 0 & 0 & 0 & 0 & 0 & 0 & 1 & 0 & 0 \\
0 & 0 & 1 & 0 & 0 & 0 & 0 & 0 & 0 & 0 & 0 & 0 & 0 & 0 & 0 & 0 \\
0 & 0 & 0 & 0 & 0 & 0 & 1 & 0 & 0 & 0 & 0 & 0 & 0 & 0 & 0 & 0 \\
0 & 0 & 0 & 0 & 0 & 0 & 0 & 0 & 0 & 0 & 1 & 0 & 0 & 0 & 0 & 0 \\
0 & 0 & 0 & 0 & 0 & 0 & 0 & 0 & 0 & 0 & 0 & 0 & 0 & 0 & 1 & 0 \\
0 & 0 & 0 & 1 & 0 & 0 & 0 & 0 & 0 & 0 & 0 & 0 & 0 & 0 & 0 & 0 \\
0 & 0 & 0 & 0 & 0 & 0 & 0 & 1 & 0 & 0 & 0 & 0 & 0 & 0 & 0 & 0 \\
0 & 0 & 0 & 0 & 0 & 0 & 0 & 0 & 0 & 0 & 0 & 1 & 0 & 0 & 0 & 0 \\
0 & 0 & 0 & 0 & 0 & 0 & 0 & 0 & 0 & 0 & 0 & 0 & 0 & 0 & 0 & 1
\end{array}\right] .
$$

Since $P_{4}^{\diamond}$ satisfies $P_{4}^{\diamond}=\left(P_{4}^{\diamond}\right)^{\prime}=\left(P_{4}^{\diamond}\right)^{-1}$, it is both an involution and a permutation matrix. $P_{4}^{\diamond}$ is the permutation matrix corresponding to transposing a $4 \times 4$ matrix $A$, e.g., reshape $\left(P_{4}^{\diamond} \operatorname{vec}(A), 4,4\right)=A^{\prime}$. We generalize this to an arbitrary size.

We introduce the function $\psi$ and verify that it is an involution that explains the action of the $P^{\diamond}$ matrices.

Definition 2.3. For $1 \leq s<n^{2}, \psi_{n}(s)=(\lceil s / n\rceil+n(s-1)) \bmod \left(n^{2}\right)$. We define $\psi_{n}\left(n^{2}\right)=n^{2}$. Denote $\psi_{n}(s)$ by $\tilde{s}$.

If the permutation action of $P_{4}^{\diamond}$ is viewed as the product of transpositions $(1)(2,5)(3,9)(4,13)(6)(7,10)(8,14)(11)(12,15)(16)$ then $\tilde{1}=1 ; \tilde{2}=5 ; \tilde{3}=9 ; \ldots$

\footnotetext{
${ }^{2}$ This operator is a derivation denoted by $a d_{A_{1}, \ldots, A_{k}}$ in [ [4], p. 37]. Each column of $M$ can be viewed as a component of the gradient of $T$.
} 
LEMMA 2.4. $\psi_{n}:\left\{1,2, \ldots, n^{2}\right\} \longrightarrow\left\{1,2, \ldots, n^{2}\right\}$ is an involution; that is, $\psi_{n}\left(\psi_{n}(s)\right)=\tilde{\tilde{s}}=s$. $\tilde{\tilde{s}}=s$.

Proof. Let $s=\alpha+\beta n, 1 \leq \alpha \leq n ; 0 \leq \beta \leq n-1$. Then $\psi_{n}(\alpha+\beta n)=(\beta+1)+(\alpha-1) n=\tilde{s}$ and

REMARK 2.5. For any $n$, we can build $P_{n}^{\diamond}$ as the sum of $n^{2} \times n^{2}$ elementary matrices, $P_{n}^{\diamond}=\sum_{r=1}^{n^{2}} E_{r, \tilde{r}}$. For $P_{n}^{\diamond}=\left(p_{i, j}\right), p_{r, c}=1 \Longleftrightarrow r=t+(s-1) n, c=s+(t-1) n=\tilde{r}$, for $1 \leq r, s \leq n$. These matrices are known [14]. Note that $\operatorname{trace}\left(P_{n}^{\diamond}\right)=n$. The $P_{n}^{\diamond}$ matrix is easily computed in MATLABC. ${ }^{3}$

Recall that for $A \in \mathcal{M}_{n}$ and $B \in \mathcal{M}_{m}$ the Kronecker product ([15, Sect.4.2]) $A \otimes B$ is given by

$$
A \otimes B=\left[\begin{array}{cccc}
a_{1,1} B & a_{1,2} B & \ldots & a_{1, n} B \\
\vdots & \vdots & & \vdots \\
a_{n, 1} B & a_{n, 2} B & \ldots & a_{n, n} B
\end{array}\right]
$$

By [15, Cor. 4.3.10] there is a unique $n^{2} \times n^{2}$ permutation matrix $P \in M_{n^{2}}(\mathbb{R})$ such that $P=P^{-1}=P^{\prime}$ and $P^{\prime}(A \otimes B) P=B \otimes A$ for every pair $(A, B)$ of $n \times n$ matrices. $P$ is given by

$$
P:=\sum_{i=1}^{n} \sum_{j=1}^{n} E_{i j} \otimes E_{i j}^{\prime} .
$$

Remark 2.6. One can confirm that $P=P^{\diamond}$. For $M \in \mathcal{M}_{n^{2}}, P M$ swaps the $s^{\text {th }}$ row with the $\tilde{s}^{\text {th }}$ row; $M P$ swaps the $t^{t h}$ column with the $\tilde{t}^{t h}$ column. From this point on $P$ will denote the permutation matrix $P^{\diamond}$ introduced above.

Proposition 2.7. Let $C=\left[A_{1}|\ldots| A_{h}\right]$ and let $C^{\top}=\left[A_{1}^{\prime}|\ldots| A_{h}^{\prime}\right]$ denote the generalized commutator of the set of transposes. Then $C^{\top}=(-1)^{\left\lceil\frac{h-1}{2}\right\rceil} C^{\prime}$.

Proof. Denote the reverse of the permutation $\pi=\{\pi(1) \pi(2) \ldots \pi(h-1) \pi(h)\}$ by $\overleftarrow{\pi}=\{\pi(h) \pi(h-1) \ldots$ $\pi(2) \pi(1)\}=\{\overleftarrow{\pi}(1) \overleftarrow{\pi}(2) \ldots \overleftarrow{\pi}(h-1) \overleftarrow{\pi}(h)\}$. Since $\operatorname{sgn}(\pi)=(-1)^{\left\lceil\frac{h-1}{2}\right\rceil} \operatorname{sgn}(\overleftarrow{\pi})$, we have $C^{\top}=\left[A_{1}^{\prime}|\ldots| A_{h}^{\prime}\right]=$ $\sum_{\pi} \operatorname{sgn}(\pi) A_{\pi(1)}^{\prime} \ldots A_{\pi(h)}^{\prime}=\left(\sum_{\pi} \operatorname{sgn}(\pi) A_{\pi(h)} A_{\pi(h-1)} \ldots A_{\pi(1)}\right)^{\prime}=\left(\sum_{\pi} \operatorname{sgn}(\pi) A_{\overleftarrow{\pi}(1)} A_{\overleftarrow{\pi}(2)} \ldots A_{\overleftarrow{\pi}(h)}\right)^{\prime}=$ $\left(\sum_{\overleftarrow{\pi}}(-1)^{\left\lceil\frac{h-1}{2}\right\rceil} \operatorname{sgn}(\overleftarrow{\pi}) A_{\overleftarrow{\pi}(1)} A_{\overleftarrow{\pi}(2)} \ldots A_{\overleftarrow{\pi}(h)}\right)^{\prime}=(-1)^{\left\lceil\frac{h-1}{2}\right\rceil} C^{\prime}$

Lemma 2.8. For $k$ odd, $P M P=-M^{\prime}$ and $m_{i, j}=-m_{\tilde{j}, \tilde{i}}$.

Proof. $P M P=-M^{\prime}$ follows from [[10],(4)]. By Remark 2.6, $m_{i, j}=-m_{\tilde{j}, \tilde{i}}$.

TheOREM 2.9. Let $k>2$ be an odd positive integer. Assume that $\mathfrak{W}=\left\{A_{1}, \ldots, A_{k}, I_{n}\right\}$ is linearly independent, and $\left\{A_{1}, \ldots, A_{k}\right\}$ generate $T$ and $M$ as above.

(i) The matrices $P M$ and $M P$ are skew-symmetric and normal.

(ii) For $n$ odd, the nullity of $T$ is greater than or equal to $k+2$.

Proof.

(i) By Lemma 2.8 $P M=-M^{\prime} P=-M^{\prime} P^{\prime}$ so $P M$ (and similarly $M P$ ) are skew-symmetric. To check normality, $(P M)^{\prime}(P M)=M^{\prime} P^{\prime} P M=M^{\prime} M=(-P M P)\left(-P M^{\prime} P^{\prime}\right)=P M M^{\prime} P^{\prime}=(P M)(P M)^{\prime}$.

\footnotetext{
${ }^{3} f=\left[1:\left(k^{2}\right)\right] ; f=(\operatorname{reshape}(f, k, k))^{\prime} ; f=f(:) ; \operatorname{Prat}=\operatorname{zeros}\left(k^{2}\right) ;$ for $j=1:\left(k^{2}\right) ; i=f i n d(f(:)==j) ; \operatorname{Pmat}(i, j)=$ $1 ;$ end
} 
(ii) If $\mathfrak{W}$ is linearly dependent, then we could replace one of the $A_{j}$ by $I$. We would then be in the case of Lemma 1.6(c) where $M \equiv 0$. This case is excluded. The eigenvalues of a skew-symmetric matrix are purely imaginary and occur in conjugate pairs. Hence, the rank of $P M$ is even. $P P M=M$ is a permutation of the rows of $P M$ so $M$ has even rank. For $n$ odd, the identity matrix $I$ is in the null space of $T$ by Remark 2.1. The null space of $T$ also contains the $k$ vectors from the generating matrices. Consequently, there is a supplementary matrix $\mathcal{S}$ that makes the dimension of the null space odd, since rank + nullity $=n$.

ExAMPLE 2.10. For $M$ generated by the five matrices in Figure $1, A_{6}$ in Figure 2 is an instance of a supplementary matrix. The generalized commutator $\left[A_{1}\left|A_{2}\right| A_{3}\left|A_{4}\right| A_{5} \mid A_{6}\right]=0$, e.g., $M v e c\left(A_{6}\right)=0$, but $\left[A_{1}\left|A_{2}\right| A_{3}\left|A_{4}\right| A_{6}\right] \neq 0$.

$$
\begin{aligned}
& A_{1}=\left[\begin{array}{rrrrr}
0 & 1 & 0 & 1 & 0 \\
0 & 0 & -1 & 0 & 0 \\
-1 & 0 & 0 & 0 & 0 \\
0 & -1 & 0 & 0 & 0 \\
0 & 0 & 0 & -1 & 0
\end{array}\right] \quad A_{2}=\left[\begin{array}{rrrrr}
-1 & 0 & 1 & 0 & -1 \\
0 & 1 & 0 & 0 & 0 \\
0 & 0 & 0 & 1 & 0 \\
-1 & 0 & -1 & 0 & 0 \\
0 & 1 & 0 & 0 & 0
\end{array}\right] \\
& A_{3}=\left[\begin{array}{rrrrr}
1 & -1 & 1 & 2 & 0 \\
1 & 0 & 1 & 1 & -1 \\
1 & 0 & -1 & 1 & 0 \\
0 & 0 & 0 & 0 & 0 \\
0 & 1 & 1 & 0 & 0
\end{array}\right] \\
& A_{4}=\left[\begin{array}{rrrrr}
-3 & 0 & 1 & 0 & 0 \\
1 & 0 & 0 & -1 & 0 \\
1 & 0 & -1 & 1 & -1 \\
1 & -1 & 1 & 0 & 0 \\
1 & 0 & 0 & 0 & 0
\end{array}\right] \quad A_{5}=\left[\begin{array}{rrrrr}
0 & -1 & 0 & 1 & 0 \\
0 & 0 & -1 & 0 & 0 \\
0 & 1 & 0 & 0 & 0 \\
-1 & 2 & 0 & 0 & -1 \\
0 & 0 & 0 & 0 & 0
\end{array}\right]
\end{aligned}
$$

Figure 1. $M$ is the matrix of $T$ generated by $A_{1}, \ldots, A_{5}$.

$$
A_{6}=\left[\begin{array}{rrrrr}
-\frac{292128568702912}{12488555496161} & \frac{140407041867250}{12488555496161} & -\frac{263825041570917}{12488555496161} & -\frac{309160140450028}{12488555496161} & 0 \\
-\frac{114784566941231}{12488555496161} & -\frac{110217311028693}{12488555496161} & -\frac{105071130066293}{12488555496161} & -\frac{350777822785063}{12488555496161} & 0 \\
\frac{10016948426257}{12488555496161} & \frac{95397472465553}{12488555496161} & \frac{23231187730969}{12488555496161} & -\frac{159185844235095}{12488555496161} & 0 \\
\frac{235605212351546}{12488555496161} & \frac{111091602885820}{12488555496161} & \frac{206801007724587}{12488555496161} & -1 & 0 \\
\frac{79564547818052}{12488555496161} & -\frac{225428073198379}{12488555496161} & -\frac{133611977232428}{12488555496161} & 0 & 0
\end{array}\right]
$$

FiguRE 2. $A_{6}$ is a supplementary matrix to $M$ generated by $A_{1}, \ldots, A_{5}$. 
Remark 2.11. Clearly $A_{6}$ is not unique since we could add any matrix in the subspace $\operatorname{Span}\left\{A_{1}, A_{2}, A_{3}\right.$, $\left.A_{4}, A_{5}, I\right\}$ to it. We demonstrate how $A_{6}$ may be calculated in Example 5.8 at the end of the paper. ${ }^{4}$

The characteristic polynomial $p_{M}(x)$ of $M_{5}$ indicates there are seven 0 roots.

$$
\begin{aligned}
p_{M}(x)= & \mathrm{x}^{25}-11192 \mathrm{x}^{23}-129557126 \mathrm{x}^{21}+1606981737331 \mathrm{x}^{19}+3245345543237967 \mathrm{x}^{17}+ \\
& -15545624559675809792 \mathrm{x}^{15}+33497503032394899259392 \mathrm{x}^{13}+267082033922488499898941440 \mathrm{x}^{11} \\
& +18200726156769208931184541696 \mathrm{x}^{9}-216569661866754313885193003335680 \mathrm{x}^{7} .
\end{aligned}
$$

3. $M$ is a commutator. The Kronecker (tensor) product ([15, Sect. 4.3]) provides

$$
\operatorname{vec}(A X B)=\left(B^{\prime} \otimes A\right) \operatorname{vec}(X) .
$$

For a sublist $\Upsilon=i_{1}<\ldots<i_{s}$ of $[1,2, \ldots, k]$ let $c(\Upsilon):=\left[A_{i_{1}}|\ldots| A_{i_{s}}\right]$, and $c([])=I$. Denote the complementary sublist of $\Upsilon$ by $\bar{\Upsilon}$. Let $\sigma(\Upsilon)$ be the sign of the permutation $\lambda:[1,2, \ldots, k+1] \longmapsto[\bar{\Upsilon},(k+1), \Upsilon]$. By [[10], 3.3 ]we can write $M$ as

$$
M:=\sum_{\Upsilon} \sigma(\Upsilon) c(\Upsilon)^{\prime} \otimes c(\bar{\Upsilon})
$$

We demonstrate this formula here for $k=1,2,3$

$$
\begin{aligned}
\mathrm{k}=1: & M_{1}=I \otimes A_{1}-A_{1}^{\prime} \otimes I \\
\mathrm{k}=2: & M_{2}=I \otimes\left[A_{1}, A_{2}\right]+\left[A_{1}, A_{2}\right]^{\prime} \otimes I+A_{1}^{\prime} \otimes A_{2}-A_{2}^{\prime} \otimes A_{1} \\
\mathrm{k}=3: & M_{3}=I \otimes\left[A_{1}, A_{2}, A_{3}\right]-\left[A_{1}, A_{2}, A_{3}\right]^{\prime} \otimes I+A_{1}^{\prime} \otimes\left[A_{3}, A_{2}\right]-\left[A_{3}, A_{2}\right]^{\prime} \otimes A_{1}+A_{2}^{\prime} \otimes\left[A_{1}, A_{3}\right]-\left[A_{1}, A_{3}\right]^{\prime} \otimes \\
& A_{2}+A_{3}^{\prime} \otimes\left[A_{2}, A_{1}\right]-\left[A_{2}, A_{1}\right]^{]^{\prime} \otimes A_{3} .}
\end{aligned}
$$

Lemma 3.1. If $k$ is even, the $k$-fold commutator has trace zero.

Proof. Let $B_{j}$ be matrices in $\mathcal{M}_{n}$. Let $\underline{C}=\left[B_{1}\left|B_{2}\right| \ldots \mid B_{k}\right]$. Consider the bijection of the symmetric group $S_{k}$ onto itself given by $\pi \longmapsto \pi^{\prime}$ where $\pi^{\prime}=\pi \cdot(12 \ldots k)$. Note that $(12 \ldots k)$ is an odd permutation (using cyclic notation) for even $k$, so $\operatorname{sgn} \pi^{\prime}=-\operatorname{sgn} \pi$. Applying $(12 \ldots k)$

$$
\left(\operatorname{sgn} \pi^{\prime}\right) B_{\pi^{\prime}(1)} \ldots B_{\pi^{\prime}(k-1)} B_{\pi^{\prime}(k)}=(-\operatorname{sgn} \pi) B_{\pi(2)} \ldots B_{\pi(k)} B_{\pi(1)} .
$$

Since $\operatorname{tr}\left(B_{i} B_{j}\right)=\operatorname{tr}\left(B_{j} B_{i}\right)$ we conclude that

$$
\operatorname{tr}\left\{\left(\operatorname{sgn} \pi^{\prime}\right) B_{\pi^{\prime}(1)} \ldots B_{\pi^{\prime}(k-1)} B_{\pi^{\prime}(k)}+(\operatorname{sgn} \pi) B_{\pi(1)} B_{\pi(2)} \ldots B_{\pi(k)}\right\}=0 .
$$

The terms of the polynomial (1.2) cancel each other in pairs. Hence, $\operatorname{tr}(\underline{C})=0$.

We recall some facts [[15],p. 250] about Kronecker (tensor) products:

- $\operatorname{tr}(A \otimes B)=\operatorname{tr}(A) \times \operatorname{tr}(B)$.

- $(B \otimes C)^{\prime}=B^{\prime} \otimes C^{\prime}$.

- $\operatorname{tr} B=\operatorname{tr} B^{\prime}$.

- $\operatorname{tr}\left(B^{\prime} \otimes C\right)=\operatorname{tr}\left(B^{\prime}\right) \operatorname{tr}(C)=\operatorname{tr}(B) \operatorname{tr}(C)$.

\footnotetext{
${ }^{4}$ In this instance, the denominator 12488555496161 in $A_{6}$ is the square root of the determinant of the principal submatrix of the first 18 rows and columns of the matrix $M_{5}$ generated by $\mathfrak{A}_{5}=\left\{A_{1}, \ldots, A_{5}\right\}$. The role of this submatrix will be discussed in Section 5.2.
} 
TheOREM 3.2. If $M$ is the matrix of $T: X \rightarrow\left[A_{1}|\ldots| A_{k} \mid X\right]$ where $A_{i}$ and $X$ are in $\mathcal{M}_{n}$, then its trace is 0 , and $M$ is a commutator.

Proof. For $k$ odd, $P M P^{-1}=-M^{\prime}$ and $\operatorname{tr} P M P^{-1}=\operatorname{tr} M P P^{-1}=\operatorname{tr} M=-\operatorname{tr} M^{\prime}=-\operatorname{tr} M$. Hence, $\operatorname{tr} M=0$.

For $k$ even $\operatorname{tr} c(\Upsilon)^{\prime} \otimes c(\bar{\Upsilon})=0$ by Lemma 3.1 whenever one of $|\Upsilon|$ or $|\bar{\Upsilon}|$ is even. The remaining terms in $M:=\sum_{\Upsilon} \sigma(\Upsilon) c(\Upsilon)^{\prime} \otimes c(\bar{\Upsilon})$ are instances where $\Upsilon$ and $\bar{\Upsilon}$ are both of odd size, in which case $\sigma(\Upsilon)=-\sigma(\bar{\Upsilon})$ Hence,

$$
\operatorname{tr}\left[\sigma(\bar{\Upsilon}) c(\bar{\Upsilon})^{\prime} \otimes c(\Upsilon)\right]=\operatorname{tr}\left[-\sigma(\Upsilon) P c(\Upsilon)^{\prime} \otimes c(\bar{\Upsilon}) P^{-1}\right]=\operatorname{tr}\left[-\sigma(\Upsilon) c(\Upsilon)^{\prime} \otimes c(\bar{\Upsilon})\right]
$$

Thus, the trace of each pair $\left[\sigma(\Upsilon) c(\Upsilon)^{\prime} \otimes c(\bar{\Upsilon})+\sigma(\bar{\Upsilon}) c(\bar{\Upsilon})^{\prime} \otimes c(\Upsilon)\right]=0$. Hence, $\operatorname{tr} M=0$, so by the result of Albert and Muckenhoupt [1] $M$ is a commutator.

All odd powers of a skew-symmetric matrix are skew-symmetric. Their traces are all zero. We generalize this here.

Theorem 3.3. For $k$ odd, the trace, $\operatorname{tr}\left(M^{2 q+1}\right)=0, q=1,2,3, \ldots$, and $M^{2 q+1}$ is a commutator.

Proof. By Lemma 2.8, a typical term in $M, m_{s, t}=-m_{\tilde{t}, \tilde{s}}$ when $k$ is odd. A typical term $h_{s, s}$ in the $(s, s)$ location of $H=M^{2 q+1}$ is given by

$$
\begin{aligned}
h_{s, s} & =\sum_{i_{1}=1}^{n} \sum_{i_{2}=1}^{n} \ldots \sum_{i_{2 q}=1}^{n} m_{s, i_{1}} m_{i_{1}, i_{2}} \ldots m_{i_{2 q-1}, i_{2 q}} m_{i_{2 q}, s} \\
& =(-1)^{2 q+1} \sum_{\tilde{i_{1}}=1}^{n} \sum_{\tilde{i_{2}}=1}^{n} \ldots \sum_{\widetilde{i_{2 q}}=1}^{n} m_{\tilde{s}, \widetilde{i_{2 q}}} m_{\widetilde{i_{2 q}} \widetilde{i_{2 q-1}}} \ldots m_{\tilde{i_{1}}, \tilde{s}}=-h_{\tilde{s}, \tilde{s}} .
\end{aligned}
$$

Hence, $h_{s, s}+h_{\tilde{s}, \tilde{s}}=0$ for all $s$, and both appear in the trace. Hence, they cancel in pairs in $\operatorname{tr}\left(M^{2 q+1}\right)$. For those values of $s$ where $s=\tilde{s}$ the terms $h_{s, s}$ are zero by this argument. Thus the trace is zero and $M^{2 q+1}$ is a commutator.

\section{Zeros in M and Hadamard products.}

REMARK 4.1. Important Bookkeeping:

(i) The unique 1 in $\operatorname{vec}\left(E_{i, j}\right)$ is the $s=i+(j-1) n^{t h}$ coefficient, and $E_{i, j}$ is the $s^{t h}$ matrix in the canonical order. $\operatorname{Mvec}\left(E_{i, j}\right)=\operatorname{vec}\left(\left[A_{1}|\ldots| A_{k} \mid E_{i, j}\right]\right)=$ the $s^{\text {th }}$ column of $M$. If $E_{i, j}$ is the $r^{t h}$ matrix in the order, $r=\alpha+\beta n, 1 \leq \alpha \leq n, 0 \leq \beta \leq n-1$, then $i=\alpha$ and $j=\beta+1$.

(ii) The column of $M$ corresponding to $E_{i, j}$ is composed of entries with terms corresponding to trails each containing a segment $\epsilon_{i, j}=\epsilon: i \rightarrow j$. The $r^{t h}$ coefficient of this column, where $r=\alpha+\beta n$, is given by trails $\alpha \longmapsto \beta+1$. For example, if $n>2$, the entries in the $3^{r d}$ row $1^{\text {st }}$ column of $M$ are trails $3 \longmapsto 1$. Equation (1.3) gives the form of these trails.

For two $4 \times 4$ matrices $A, B, \mathfrak{A}=\{A, B\}$, the $2^{\text {nd }}$ column of $M_{\mathfrak{A}}$ is given by Figure 3 . We interpret this column of data by including the element $\epsilon_{21}$ from $E_{2,1}$, the second matrix in the canonical ordering. The last term becomes $\left(a_{14} b_{42}-a_{42} b_{14}\right) \epsilon_{21}=b_{42} \epsilon_{2,1} a_{14}-a_{42} \epsilon_{2,1} b_{14}$ which represent two trails $4 \rightarrow 2 \rightarrow 1 \rightarrow 4$. Similarly, the term in the $2^{\text {nd }}$ last position represents two trails $3 \rightarrow 2 \rightarrow 1 \rightarrow 4$ with the help of the $\epsilon_{2,1}$. Henceforth, we consider entries of $M$ as a sum of terms with associated trails with the $\epsilon$ included. We explain the reason for a zero in the fifth position here. 


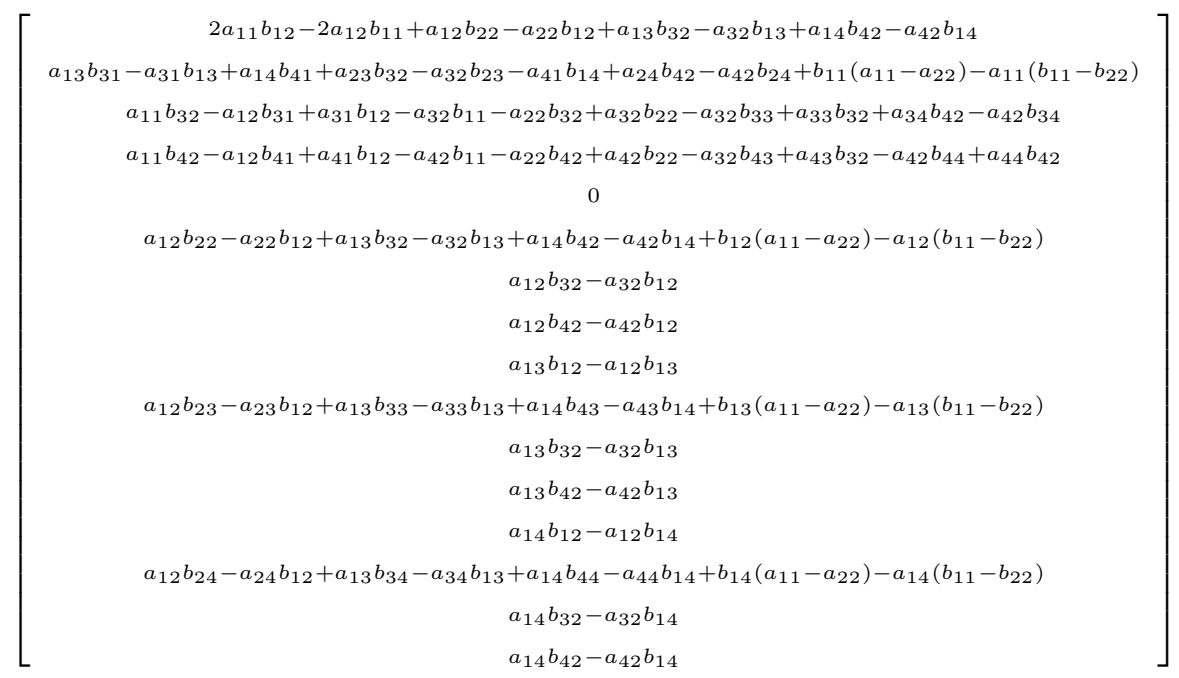

Figure 3. The $2^{\text {nd }}$ column of $M_{\mathfrak{A}}$.

Lemma 4.2. Let $M$ be the matrix of the linear operator $T_{A, B}$ generated by two matrices. For any such that $s \neq \tilde{s}$ the $[s, \tilde{s}]$ entries of $M, m_{s, \tilde{s}}=0$.

Proof. Using the notation of Lemma 2.4, $s=\alpha+\beta n, 0 \leq \alpha \leq n-1 ; 0 \leq \beta \leq n-1$. By Remark 4.1 (ii), every $m_{s, \tilde{s}}$ term has one of two forms: $a_{\alpha, \beta+1} \epsilon_{\beta+1, \alpha} b_{\alpha, \beta+1}$ or $b_{\alpha, \beta+1} \epsilon_{\beta+1, \alpha} a_{\alpha, \beta+1}$. These have opposite parity and cancel in pairs.

The following Lemma is easily verified.

Lemma 4.3. Given a permutation matrix $P$, and $H_{1}, H_{2}$ all in $\mathcal{M}_{n}$, then the Hadamard product $H_{1} \circ H_{2}=$ $0 \Longleftrightarrow H_{1} P \circ H_{2} P=0 \Longleftrightarrow P H_{1} \circ P H_{2}=0$.

TheOREm 4.4. Let $M$ be the matrix of $T$ for $k$ odd. Then, the Hadamard product $M^{2 q+1} \circ P^{\diamond}=O, q=$ $0,1,2,3 \ldots$, where $P^{\diamond}$ is defined by (2.7). Hence, the entries of $M^{2 q+1}$ corresponding to the ones of $P^{\diamond}$ are zero.

Proof. Case q=0: Since $P^{\diamond} M$ is skew symmetric, it has zeros down the diagonal. Hence, $P^{\diamond} M \circ I=0$. By Lemma 4.3 above, $P^{\diamond} P^{\diamond} M \circ P^{\diamond} I=0 \Rightarrow M \circ P^{\diamond}=0$.

Case $q>0$ : By Lemma 2.8, $m_{i, j}=-m_{\tilde{j}, \tilde{i}}$. A typical term $h_{s, \tilde{s}}$ in the $(s, \tilde{s})$ location of $H=M^{2 q+1}$ is given by

$$
\begin{aligned}
& h_{s, \tilde{s}}=\sum_{i_{1}=1}^{n} \sum_{i_{2}=1}^{n} \ldots \sum_{i_{2 q}=1}^{n} m_{s, i_{1}} m_{i_{1}, i_{2}} \ldots m_{i_{2 q}, \tilde{s}} \\
& =(-1)^{2 q+1} \sum_{\tilde{i_{1}}=1}^{n} \sum_{\tilde{i_{2}}=1}^{n} \ldots \sum_{\widetilde{i_{2 q}}=1}^{n} m_{s, \widetilde{i_{2 q}}} m_{\widetilde{i_{2 q}}} \widetilde{i_{2 q-1}} \ldots m_{\tilde{i_{1}, \tilde{s}}}=-h_{s, \tilde{s}} .
\end{aligned}
$$

Hence, $h_{s, \tilde{s}}=0$ for all $s$, so $M^{2 q+1} \circ P^{\diamond}=O$ since every nonzero element of $P^{\diamond}$ is found at the $(s, \tilde{s})$ location by Remark (2.5). 
Denote the Moore-Penrose inverse of $M$ by $M^{+}$. Based on experimental evidence, we conjecture that $\left(M^{+}\right)^{2 q+1} \circ P^{\diamond}=O$ for $q=1, \ldots$

\section{The Dimension of the Null Space of $\mathbf{T}$.}

5.1. The Reduced Row Echelon Form: RREF. Given $L \in \mathcal{M}_{m}$ of rank $(m-q)$, let $L_{r r e f}$ denote its unique RREF. It can be written, up to some permutation of columns, as

$$
L_{\text {rref }}=\left[\begin{array}{cc}
I_{m-q} & \tilde{U} \\
O & O
\end{array}\right]
$$

where the size of $\tilde{U}$ is $(m-q) \times q$. Note that if the first $m-q$ columns of $L$ are linearly independent, then no column permutations are required in the RREF. Construct the array $U=\left[\begin{array}{c}\tilde{U} \\ -I\end{array}\right]$ whose columns are a basis of the null space of $L$. The transpose of the $j^{\text {th }}$ column of $U_{\text {rref }}$ is a row whose entries are $\left[u_{1, j}, \ldots, u_{m-q, j}, \ldots,-1,0 \ldots 0\right]$ and the last $q$ contain $(q-1) 0^{\prime} s$ together with a unique -1 , in some order. Call vectors of this type right-ended. Matrices reshaped from right-ended vectors of size $n^{2}$ are called right-ended matrices. This motivates the following.

5.2. Generic Right-Ended Matrices (GREMs). A set of elements in $\mathbb{R}$ is called generic if it is algebraically independent over $\mathbb{Q}$. Choose matrices $\mathfrak{A}=\left\{A_{1}, \ldots, A_{k}\right\}$ built from $k n^{2}$ distinct algebraically independent generic elements.

$$
\text { For } \mathfrak{A}=\left\{A_{1}, \ldots, A_{k}\right\} \text {, let } V_{\mathfrak{A}}=\left[\operatorname{vec}\left(A_{1}\right), \ldots, \operatorname{vec}\left(A_{k}\right)\right]=\left[\begin{array}{c}
A_{(n-k) k}^{b} \\
A_{k \times k}^{\natural}
\end{array}\right] \text {. }
$$

By Remark 2.1, $M V_{\mathfrak{A}}=0$, for $M$ generated by $\mathfrak{A}$. The determinant $\left|A^{\natural}\right|=\xi \neq 0$ because it is a polynomial of degree $k$ in algebraically independent elements. Thus, $A^{\natural}$ is nonsingular with inverse $\left(A^{\natural}\right)^{-1}=\left[\gamma_{i j}\right]$. The columns of $-V_{\mathfrak{A}}\left(A^{\natural}\right)^{-1}$ are right-ended, so we reshape them into right-ended matrices $A_{j}^{\Gamma}, 1 \leq j \leq k$.

$$
\text { Let } A^{\Gamma}=\left[\operatorname{vec}\left(A_{1}^{\Gamma}\right), \ldots, \operatorname{vec}\left(A_{k}^{\Gamma}\right)\right]=-V_{\mathfrak{A}}\left(A^{\natural}\right)^{-1}, \text { where } A_{j}^{\Gamma}:=-\sum_{i=1}^{k} \gamma_{i j} A_{i} \text {. }
$$

By $([10],(3)),\left[A_{1}^{\Gamma}|\ldots| A_{k}^{\Gamma} \mid X\right]=-(\xi)^{-1} \times\left[A_{1}|\ldots| A_{k} \mid X\right]$, so both or neither term is 0 . We note that the entries of the $A_{i}^{\Gamma}$ matrices are still algebraically independent, since they are polynomials in the original set of generic elements with a common denominator of products of $\xi=\left|A^{\natural}\right|$.

Proposition 5.1. Let $T^{\mathfrak{A}}$ and $T^{\Gamma}$ denote the linear transformations generated by $\mathfrak{A}=\left\{A_{1}, A_{2}, \ldots, A_{k}\right\}$ and $\mathfrak{A}^{\Gamma}=\left\{A_{1}^{\Gamma}, A_{2}^{\Gamma}, \ldots, A_{k}^{\Gamma}\right\}$ respectively. Then $T^{\Gamma}(X)=-(\xi)^{-1} T^{\mathfrak{A}}(X)$ and the null spaces of both operators are the same.

Null spaces do not change when we require the generic matrices to be right-ended. From this point onward, we constrain the input matrices to be right-ended and call them Generic Right-Ended Matrices or GREMs. Denote the vector $\operatorname{vec}\left(A_{j}\right)$ with its right end cut off by $\breve{A}_{j}$. Denote a matrix whose columns are "cup" vectors by a "tilde" $\tilde{A}=\left[\breve{A}_{1}, \ldots, \breve{A}_{k}\right]$. In this $\operatorname{case}^{5}$ we write $(5.11)$ as

$$
A_{\Gamma}=\left[\operatorname{vec}\left(A_{1}^{\Gamma}\right), \ldots, \operatorname{vec}\left(A_{k}^{\Gamma}\right)\right]=\left[\begin{array}{l}
\tilde{A}_{(n-k) \times k} \\
-I_{k}
\end{array}\right]
$$

\footnotetext{
${ }^{5} \operatorname{In}$ MATLAB $(C), A_{\Gamma}=-\left[\operatorname{flipud}\left(\operatorname{flip} \operatorname{lr}\left(\operatorname{rref}\left(\operatorname{fliplr}\left(A_{\mathfrak{A}}^{\prime}\right)\right)\right)\right)\right]^{\prime}$.
} 
We modify the new right-ended matrices by prescribing the right ends of the canonical rows in Definition 1.5 according to whether $k$ is even or odd. The last end of Case ${ }_{1}$ is deleted from $\mathrm{Case}_{2}$ in order to provide space for the identity matrix in the null space of $M$.

$$
\begin{aligned}
& \text { Case }_{1}: k \text { even: } \begin{array}{llll}
{[\ldots-1,0,0 \ldots, 0,0,0]} & {[\ldots 0,-1,0 \ldots, 0,0,0]} & {[\ldots 0,0,-1 \ldots, 0,0,0] \cdots} \\
{[\ldots 0,0,0 \ldots,-1,0,0]} & {[\ldots 0,0,0 \ldots, 0,-1,0]} & {[\ldots 0,0,0 \ldots, 0,0,-1] .}
\end{array} \\
& \text { Case }_{2}: k \text { odd: }[\ldots-1,0,0 \ldots, 0,0,0] \quad[\ldots 0,-1,0 \ldots, 0,0,0] \quad \cdots \\
& {[\ldots 0,0 \ldots,-1,0,0,0] \quad[\ldots 0,0 \ldots, 0,-1,0,0] \quad[\ldots 0,0 \ldots, 0,0,-1,0] .}
\end{aligned}
$$

Taking vec of a right-ended matrix gives a transposed right-ended vector. The dimension of the null space of $T$ generated by $\mathfrak{A}$ or $\mathfrak{A}^{\Gamma}$ is denoted by $\nu_{0}(n, k)$. Partition $M_{\Gamma}$, the matrix of $T$ generated by $\mathfrak{A}^{\Gamma}$, where the principal submatrix $M_{1,1}$ is square and has the largest possible rank that the first square block can achieve.

$$
M_{\Gamma}=\left[\begin{array}{ll}
M_{1,1} & M_{1,2} \\
M_{2,1} & M_{2,2}
\end{array}\right] \quad M_{1}=\left[\begin{array}{l}
M_{1,1} \\
M_{2,1}
\end{array}\right] \quad M_{2}=\left[\begin{array}{c}
M_{1,2} \\
M_{2,2}
\end{array}\right]
$$

Conjecture 1 was introduced in [10]. Matt Brassil verified, in his dissertation [6] that over a large set of finite fields the conjecture is true in the case $k$ even using graphical techniques. This has been refined in a joint paper with Zinovy Reichstein [7].

Conjecture 1. For almost all choices of the generic matrices $A_{i} 1 \leq i \leq k$

(i) $\nu_{0}(n, k):=k$ if $k$ is even

(ii) $\nu_{0}(n, k):=k+1$ if $k$ is odd and $n$ is even

(iii) $\nu_{0}(n, k):=k+2$ if $k$ is odd and $n$ is odd.

REMARK 5.2. Conjecture 1 was originally formulated on experimental evidence. Upon revisiting this evidence and performing further corroborative trials, we introduce a new conjecture that prescribes the rank of $M_{1,1}$. With this addition, we show that supplementary matrices, which heretofore were mysterious, can be explained using the Moore-Penrose inverse. This is a main point of this paper. It also avoids consideration of the need to treat the permutation of RREF columns mentioned in Section 5.1.

Conjecture 2. For almost all choices of GREM's $A_{i}^{\Gamma} 1 \leq i \leq k, k>1$

(i) $\nu_{0}(n, k):=k$ if $k$ is even. $M_{1,1}$ has rank $n^{2}-k$.

(ii) $\nu_{0}(n, k):=k+1$ if $k$ is odd and $n$ is even. $M_{1,1}$ has rank $n^{2}-(k+1)$.

(iii) $\nu_{0}(n, k):=k+2$ if $k$ is odd and $n$ is odd. $M_{1,1}$ has rank $n^{2}-(k+2)$.

We justify the sizes of the null space in Conjecture 2 as follows.

(i) By Lemma 1.6, the $k$ generating matrices are a basis of the null space of $M$.

(ii) By Remark 2.1, $T(I)=0$ when $k$ is odd. The last end in Case ${ }_{1}$ is absent in Case e $_{2}$ to leave space for the Identity matrix in the null space of $M$.

(iii) By Theorem 2.9 (ii), when $k$ and $n$ are both odd, there must be at least one supplementary matrix in the null space of $T$. Conjecture 2 indicates that for generic $A_{j}^{\Gamma}$ there is exactly one supplementary matrix $\mathcal{S}$, modulo addition of a matrix in the span of

$$
\mathfrak{W}=\left\{A_{1}, \ldots, A_{k}, I_{n}\right\} .
$$


Electronic Journal of Linear Algebra, ISSN 1081-3810

A publication of the International Linear Algebra Society

Volume 37, pp. 598-612, September 2021.

Every matrix $L$ in $\mathcal{M}_{n}$ has a full rank factorization $L=F G$, where the columns of $F$ are a basis of the range space of $L$, and $G$ is uniquely determined by $F$. Conjecture 2 specifies that $F=M_{1}$, using the notation of (5.13).

Let $H_{a: b, c: d}$ denote the submatrix of $H$ with rows $a$ to $b$ and columns $c$ to $d$. The submatrix of columns $c, \ldots, d$ and all rows of $H$ is denoted by $H_{\bullet, c: d}$. By $[5,16]$, for a full column rank matrix $F$, the Moore-Penrose inverse is

$$
F^{+}=\left(F^{\prime} F\right)^{-1} F^{\prime}
$$

5.2.1. Consequences of Conjecture 2 when $\boldsymbol{k}$ is even. The elegance of the structure of $M$ is explained once the Moore-Penrose inverse is introduced. Using the notation of (5.13), the RREF for the case $k$ even can be found without the usual computation, once $\mathfrak{A}^{\Gamma}=\left\{A_{1}^{\Gamma}, \ldots, A_{k}^{\Gamma}\right\}$ is given.

ThEOREM 5.3. Assume Conjecture 2 is true. For $k$ even and $M$ generated by $\mathfrak{A}^{\Gamma}$, the arrangement of the RREF $M_{\text {rref }}$ and the nonsingular matrix $Q$ such that $Q M=M_{\text {rref }}$ are fixed.

$$
M_{\text {rref }}=\left[\begin{array}{cc}
I & \tilde{A} \\
O & O
\end{array}\right] \quad Q=\left[\begin{array}{ll}
M_{1,1}^{-1} & O \\
M_{2,1} M_{1,1}^{-1} & -I
\end{array}\right] \quad Q^{-1}=\left[\begin{array}{ll}
M_{1,1} & O \\
M_{2,1} & -I
\end{array}\right]
$$

Following the notation of (5.10) $\tilde{U} \equiv \tilde{A}=\left(M_{1,1}\right)^{-1} M_{1,2}$. A full rank factorization of $M$ is given by $F=M_{1}=\left[\begin{array}{c}M_{1,1} \\ M_{1,1} \tilde{A}\end{array}\right], G=\left[I_{n^{2}-k}, \tilde{A}\right]$ where $F^{+} M=G$ and $F^{+} M_{2}=F^{+} M_{\bullet,\left(n^{2}-k+1: n^{2}\right)}=\tilde{A}$.

Proof. By the uniqueness of the RREF and Conjecture $2, \tilde{U} \equiv \tilde{A}$ since the generating matrices $\mathfrak{A}^{\Gamma}$ are right ended and are a basis of the $k$ dimensional null space of $M$ and $M_{1,1}$ is invertible. Using the notation of $(5.12), M A_{\Gamma}=\left[M_{1}, M_{2}\right]\left[\begin{array}{c}\tilde{A} \\ -I\end{array}\right]=M_{1} \tilde{A}-M_{2}=O$. Hence, $M_{1} \tilde{A}=M_{2}, M_{1,2}=M_{1,1} \tilde{A} \Longleftrightarrow \tilde{A}=$ $\left(M_{1,1}\right)^{-1} M_{1,2}$, and $M_{2,2}=M_{2,1} \tilde{A}=M_{2,1}\left(M_{1,1}\right)^{-1} M_{1,2}$.

$$
\begin{gathered}
Q M=\left[\begin{array}{lr}
M_{1,1}^{-1} & O \\
M_{2,1} M_{1,1}^{-1} & -I
\end{array}\right]\left[\begin{array}{ll}
M_{1,1} & M_{1,2} \\
M_{2,1} & M_{2,2}
\end{array}\right]= \\
{\left[\begin{array}{lr}
M_{1,1}^{-1} M_{1,1} & M_{1,1}^{-1} M_{1,2} \\
M_{2,1}-M_{2,1} & M_{2,1} M_{1,1}^{-1} M_{1,2}-M_{2,2}
\end{array}\right]=\left[\begin{array}{cc}
I_{n^{2}-k} & \tilde{U} \\
O & O
\end{array}\right]=M_{r r e f}}
\end{gathered}
$$

Clearly $M=F G$ and $F^{+} F=I$, so $F^{+} M=F^{+} F G=G$. Thus $F^{+} M_{2}=\tilde{A}$.

TheOREM 5.4. Assume Conjecture 2 is true. If $H \in \mathcal{M}_{n^{2}}$ is a matrix of rank $\left(n^{2}-k\right)$ where $k$ is even, then $H$ is equivalent to a k-commutator matrix.

Proof. Without loss of generality, permuting rows and columns if necessary to get an equivalent matrix, partition $H=\left[\begin{array}{ll}H_{1,1} & H_{1,2} \\ H_{2,1} & H_{2,2}\end{array}\right]$ so that $H_{1,1}$ is nonsingular of size $n^{2}-k$. Write the unique reduced row echelon form of $H$ as $H_{\text {rref }}=\left[\begin{array}{cc}I_{n^{2}-k} & \tilde{A}^{H} \\ O & O_{k}\end{array}\right]$. Use $\tilde{A}^{H}$ to build $M^{\star}$, a k-commutator matrix, as in Theorem 5.3 , where $Q M^{\star}=M_{r \text { ref }}^{\star}=H_{\text {rref }}$. Define $Q^{H}=\left[\begin{array}{cc}H_{1,1}^{-1} & O \\ H_{2,1} H_{1,1}^{-1} & -I\end{array}\right] ;$ so that $Q^{H} H=H_{\text {rref }}=Q M^{\star}$. Hence, $M^{\star}=Q^{-1} Q^{H} H$ so $H$ and $M^{\star}$ are equivalent. 
5.2.2. Consequences of Conjecture 2 when $k$ is odd and $n$ even. As a consequence of the Amitsur-Levitski Theorem [2], we require that $k<2 n-1$ throughout the following. The $A_{j}^{\Gamma}$ are GREMs with ends described by Case ${ }_{2}$ of Conjecture 2. Let $\breve{I}$ denote vec $(I)$ minus its end, and $\tilde{A}^{\Gamma}$ the matrix given in (5.12) obtained from the generating matrices, using the Case $e_{2}$ arrangement. If $n<k<2 n-1 \operatorname{vec}(-I)$ is improperly right-ended according to $\mathrm{Case}_{2}$, since it has $2(-1)$ 's in its end.

We introduce the matrix $\mathcal{C}$ for the Theorem below to deal with the case $k>n$, where we define $\mathcal{C}=-I-A_{n-k}^{\Gamma}$. This is right-ended and in the null space of $M$. When $k \leq n$, we set $\mathcal{C}=-I$.

Theorem 5.5. Assume Conjecture 2 is true. For $k$ odd and $n$ even and $M$ generated by $\mathfrak{A}^{\Gamma}=\left\{A_{1}^{\Gamma}, \ldots\right.$, $\left.A_{k}^{\Gamma}\right\}$ then the arrangement of the RREF $M_{\text {rref }}$ and the nonsingular matrix $Q$ such that $Q M=M_{\text {rref }}$ are fixed. Let $\tilde{U}=\left[\tilde{A^{\Gamma}}, \tilde{\mathcal{C}}\right]$. $\tilde{U}=\left(M_{1,1}\right)^{-1} M_{1,2}$. A full rank factorization of $M$ is given by $F=M_{1}, G=$ $\left[I_{n^{2}-(k+1)}, \tilde{U}\right]$ where $F^{+} M=G$ and $F^{+} M_{2}=F^{+} M_{\bullet,\left(n^{2}-k: n^{2}\right)}=\tilde{U}$.

Proof. By Remark 2.1, when we extend the set $\mathfrak{A}^{\Gamma}$ by $-I$ to give a new set $\mathfrak{A}_{k+1}^{\Gamma}=\left\{A_{1}^{\Gamma}, \ldots, A_{k}^{\Gamma},-I\right\}$, then both sets are right-ended and produce the same $M$. The proof follows directly from Theorem 5.3 since $(k+1)$ is even.

5.2.3. Consequences of Conjecture 2 when $k$ and $n$ are both odd. By Conjecture 2, there is a unique supplementary matrix $\mathcal{S}$ modulo matrices in span $\mathfrak{W}(5.14)$. Let $\breve{\mathcal{S}}$ be $\operatorname{vec}(\mathcal{S})$ minus its end. A reason for introducing Conjecture 2 was to ensure the RREF of $M$ will find $\breve{\mathcal{S}}$ in column $n^{2}-(k+1)$. We define 3 cases for $\mathcal{C}$

$$
\left\{\begin{array}{ll}
k<n & \mathcal{C}=-I \\
k=n & \mathcal{C}=-I-\mathcal{S} \\
k>n & \mathcal{C}=-I-A_{n-k}^{\Gamma}
\end{array} .\right.
$$

Set $U=\left[\operatorname{vec}(\mathcal{S}), A^{\Gamma}, \operatorname{vec}(\mathcal{C})\right]$, and $\tilde{U}=\left[\breve{\mathcal{S}}, \tilde{A}^{\Gamma}, \breve{\mathcal{C}}\right] \cdot M_{1,1}$ is nonsingular of size $n^{2}-(k+2)$. Define $B=$ $M_{2,1} M_{1,1}^{-1} \cdot\left(B^{\prime} B\right)$ is positive semidefinite, so $\left(I+B^{\prime} B\right)$ is positive definite. The determinant of $\left(I-B^{\prime} B B^{\prime} B\right)$ can be expressed in terms of quotients of polynomials in the generic elements introduced earlier. The probability of this determinant being zero is 0 , so we may consider $\left(I-B^{\prime} B B^{\prime} B\right)$ to be nonsingular. Thus $\left(I-B^{\prime} B\right)\left(I+B^{\prime} B\right)=\left(I-B^{\prime} B B^{\prime} B\right) \Longrightarrow$

$\left(I+B^{\prime} B\right)^{-1}=\left(I-B^{\prime} B B^{\prime} B\right)^{-1}\left(I-B^{\prime} B\right) . \quad F=M_{1} \Longrightarrow M_{1}^{\prime}=\left[M_{1,1}^{\prime}, M_{2,1}^{\prime}\right]$.

$$
\begin{array}{r}
F^{+}=\left(M_{1}^{\prime} M_{1}\right)^{-1} M_{1}^{\prime}=\left(M_{1,1}^{\prime} M_{1,1}+M_{2,1}^{\prime} M_{2,1}\right)^{-1} M_{1}^{\prime} \\
=\left(M_{1,1}^{\prime} M_{1,1}+M_{1,1}^{\prime} B^{\prime} B M_{1,1}\right)^{-1} M_{1}^{\prime}=\left(M_{1,1}^{\prime}\left(I+B^{\prime} B\right) M_{1,1}\right)^{-1}\left[M_{1,1}^{\prime}, M_{2,1}^{\prime}\right] \\
=M_{1,1}^{-1}\left[\left(I-B^{\prime} B B^{\prime} B\right)^{-1}\left(I-B^{\prime} B\right),\left(I-B^{\prime} B B^{\prime} B\right)^{-1}\left(B^{\prime}-B^{\prime} B B^{\prime}\right)\right] .
\end{array}
$$

This gives an explicit description of $F^{+}$, the Moore-Penrose inverse of $F$.

Theorem 5.6. Assume Conjecture 2 is true. For $k$ odd, $n$ odd and $M$ generated by $\mathfrak{A}^{\Gamma}$ with Case 2 ends, then $F=M_{1}=M_{\bullet, 1:\left(n^{2}-k-2\right)}$ and $G=\left[I_{n^{2}-(k+2)}, \tilde{U}\right]$ is a full rank factorization of $M$. $M_{\text {rref }}=$

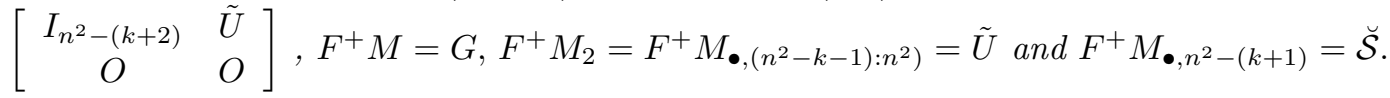

Proof. Since $M U=0$ the proof that $M=F G$ is identical to that of Theorem 5.3. If $k=n$ the second last diagonal 1 of $I$ coincides with the -1 in the right-end of $\mathcal{S}$ so $\breve{\mathcal{C}}=-\breve{I}-\breve{\mathcal{S}}$ is the only possibility for the final column. As in Theorem 5.3 we verify $F^{+} M=G, F^{+} M_{2}=\tilde{U}$. Hence, we have $\breve{\mathcal{S}}=F^{+} M_{\bullet}, n^{2}-(k+1)$. 
Thus, we can explicitly describe a supplementary matrix $\mathcal{S}$ in terms of the entries of $M$ by the description of $F^{+}$above and the last item of the Theorem. Moreover, we have shown by Theorem 5.3 and the above, that the entries of $\mathcal{S}$ are quotients of polynomials in the entries of the original $A_{j}$ matrices. We provide two final examples.

EXAMPLE 5.7. The matrix $M_{3}$ is generated by the three right- ended matrices:

$$
\begin{aligned}
& {\left[\begin{array}{rrr}
0 & -2 & 0 \\
2 & 0 & 0 \\
-4 & -1 & 0
\end{array}\right] \quad\left[\begin{array}{rrr}
4 & 0 & -1 \\
4 & 0 & 0 \\
-1 & 0 & 0
\end{array}\right] \quad\left[\begin{array}{rrr}
-4 & 3 & 0 \\
5 & 0 & -1 \\
0 & 0 & 0
\end{array}\right] .} \\
& M_{3}=\left[\begin{array}{rrrrrrrrr}
0 & -24 & -31 & 36 & 7 & 4 & -65 & -12 & -7 \\
-36 & -36 & -16 & 0 & -4 & -8 & -272 & -36 & 40 \\
65 & -112 & -188 & 272 & 5 & -16 & 0 & -4 & -70 \\
24 & 0 & -16 & 36 & -8 & -8 & 112 & 12 & -16 \\
-7 & 8 & 9 & 4 & 0 & -28 & -5 & 80 & 7 \\
12 & -12 & -4 & 36 & -80 & -80 & 4 & 0 & 68 \\
31 & 16 & 0 & 16 & -9 & 0 & 188 & 4 & -22 \\
-4 & 8 & 0 & 8 & 28 & 0 & 16 & 80 & -24 \\
7 & 16 & 22 & -40 & -7 & 24 & 70 & -68 & 0
\end{array}\right] .
\end{aligned}
$$

The Moore-Penrose inverse is computed as in Theorem 5.6:

$$
\begin{aligned}
& F^{+}=\left[\begin{array}{rrrrr}
-\frac{527711}{522335175} & -\frac{1732708}{522335175} & \frac{33646}{27491325} & \frac{1779316}{522335175} & \\
-\frac{6979654}{6790357275} & -\frac{209532799}{13580714550} & \frac{396719}{357387225} & \frac{46793171}{27161429100} & \\
-\frac{1848148}{452690485} & \frac{2502901}{452690485} & -\frac{152127}{23825815} & \frac{1234148}{452690485} & \\
-\frac{5596264}{2263452425} & -\frac{15138993}{9053809700} & -\frac{88746}{119129075} & \frac{9799093}{4526904850}
\end{array}\right. \\
& \begin{array}{rrrrr}
-\frac{8938289}{522335175} & \frac{232372}{20893407} & \frac{4367146}{522335175} & -\frac{3481512}{174111725} & \frac{378640}{20893407} \\
\frac{104114429}{6790357275} & -\frac{36462259}{1086457164} & \frac{11336894}{6790357275} & \frac{125054539}{4526904850} & -\frac{3885391}{271614291}
\end{array} \\
& \frac{3429083}{452690485} \quad \frac{4214407}{90538097} \quad \frac{434413}{452690485} \quad-\frac{4275158}{452690485} \quad-\frac{316187}{90538097}
\end{aligned}
$$

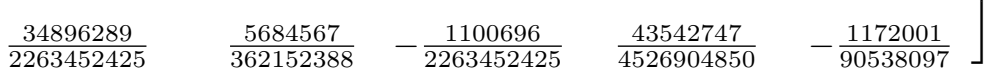

Rank $M_{1,1}=4,\left|M_{1,1}\right|=(4 \times 39)^{2}$, and the supplementary matrix is

$$
\mathcal{S}=\left[\begin{array}{rrr}
-\frac{5}{3} & -\frac{12}{13} & 0 \\
\frac{140}{39} & -1 & 0 \\
-\frac{53}{13} & 0 & 0
\end{array}\right] \text {. }
$$


EXAMPLE 5.8. We return to Example 2.10. Using exact arithmetic ${ }^{6}$, we find that the particular supplementary matrix $A_{6}(2.8)$ is precisely equal to the reshape of $M_{1}^{+} M(1: 18,19)=\left[\left(M_{1}^{\prime} M_{1}\right)^{-1} M_{1}^{\prime} M(1: 18,19)\right]$ with the tail $\left[\begin{array}{lllllll}-1 & 0 & 0 & 0 & 0 & 0 & 0\end{array}\right]^{\prime}$ appended, where $M$ is the matrix generated by the 5 given matrices. Each column of $M_{1}$ is equal to $\operatorname{vec}\left(\left[A_{1}\left|A_{2}\right| \ldots\left|A_{5}\right| E_{i}\right]\right)$ for the first 18 elementary matrices. Using the Moore-Penrose inverse, $A_{6}$ is expressed algebraically by the entries of the $5 A_{j}$ matrices.

Summary. The purpose of this paper was to explore the properties of the generalized commutator and to explain the mysterious supplementary matrix. Conjecture 2 still requires verification, but it is based on an aggregate of experimental evidence. The role of the Moore-Penrose inverse is felicitous.

Acknowledgments. The author is grateful to John Dixon and the anonymous referee for their generous advice regarding this paper. An invitation to Queens University by Ram Murty led to this research.

\section{REFERENCES}

[1] A.A. Albert and B. Muckenhoupt. On matrices of trace zero. Michigan Math. J., 4(1):1-3, 1957.

[2] A.S. Amitsur and J. Levitzki. Minimal identities for algebras. Proc. Am. Math. Soc. 1(4):448-463, 1950.

[3] A.S. Amitsur and J. Levitzki. Remarks on minimal identities for algebras. Proc. Am. Math. Soc. 2(2):320-327, 1951.

[4] J.A. de Azcarraga and J.M. Izquierdo. n-ary algebras: A review with applications. J. Phys. A Math. Theor. 43(29):293001, 2010 .

[5] A. Ben-Israel and T. Greville. Generalized Inverses: Theory and Applications. Springer Science \& Business Media. Vol. $15,2003$.

[6] M. Brassil. A graph-theoretic approach to a conjecture of Dixon and Pressman. Electronic Theses and Dissertations (ETDs) 2008+, 2020. https://open.library.ubc.ca/collections/ubctheses/24/items/1.0394102.

[7] M. Brassil and Z. Reichstein. A graph-theoretic approach to a conjecture of Dixon and Pressman. arXiv preprint arXiv:2010.04679. To appear Israel Journal of Mathematics.

[8] M. Bremner. Identities for the ternary commutator. J. Algebra 206(2):615-623, 1998.

[9] C. Devchand, D. Fairlie, J. Nuyts, and G. Weingart. Ternutator identities. J. Phys. A Math. Theor. 42(47):475209, 2009.

[10] J.D. Dixon and I.S. Pressman. Generalized commutators and a problem related to the Amitsur-Levitzki Theorem, Linear Multilinear Algebra 66(11):2199-2207, 2018.

[11] M.P. Drazin. On diagonable and normal matrices. Quar. J. Math. 2(1):189-998, 1951.

[12] A.S. Dzhumadil'daev. N-commutators. Commentarii Mathematici Helvetici 79(3):516-553, 2004.

[13] E. Formanek. The Polynomial Identities and Invariants of $n \times n$ Matrices. American Mathematical Soc. Vol. $78,1991$.

[14] H.V. Henderson and S.R. Searle. The vec-permutation matrix, the vec operator and Kronecker products: A review. Linear Multilinear Algebra 9(4):271-288, 1981.

[15] R.A. Horn and C.R. Johnson. Topics in Matrix Analysis. Cambridge University Press, 1991.

[16] R. Piziak and P.L. Odell. Full Rank Factorization of Matrices. Math. Assoc. Am. Math. Mag. 72(3):193-201, 1999.

[17] L.H. Rowen. Standard polynomials in matrix algebras. Trans. Am. Math. Soc. 190:253-284, 1974.

[18] R.G. Swan. An application of graph theory to algebra. Proc. Am. Math. Soc. 14(3):367-373, 1963.

\footnotetext{
${ }^{6}$ not the less accurate "pinv" MATLAB (c) function
} 